\title{
Black Hole: A New Operator for Gravitational Search Algorithm
}

\author{
Mohammad Doraghinejad \\ Department of Electrical Engineering, Shahid Bahonar University of Kerman, P.O. Box 76169-133 \\ Kerman, Iran \\ Hossein Nezamabadi-pour \\ Department of Electrical Engineering, Shahid Bahonar University of Kerman, P.O. Box 76169-133 \\ Kerman, Iran \\ nezam@uk.ac.ir,www.uk.ac.ir
}

Received 15 March 2013; Accepted 26 August 2014

\begin{abstract}
Inspiring by nature have motivated many researchers in many fields of sciences and engineering. The Gravitational search algorithm (GSA) is a recent created metaheuristic algorithm by using law of gravity and mass interactions. In this paper, a new operator inspired by some of the characteristics of the black hole as an astronomy phenomenon for GSA is presented. When a star is converted to a black hole under situations, it has the extremely strong gravity that prevents anything to escape from, and the objects that are closed to the black hole, experience very strong force called tidal force which it causes to collapse them to the black hole. We propose a new operator using these features and hybridize it with GSA (BH-GSA) in order to prevent facing the premature convergence and to improve the abilities of GSA in exploration and exploitation. The proposed algorithm is applied to two sets of standard benchmark functions. The first set includes 23 standard benchmark functions and in this set the performance of the proposed algorithm is compared with the standard GSA, the disruption GSA, the particle swarm optimization (PSO), and the real genetic algorithm (GA). The second set contains the CEC 2005 benchmark functions. In this set, we compare the BH-GSA with some well-known metaheuristic algorithms. The obtained results and comparing with the competing algorithms prove that the BH-GSA has merit in the field of continuous space optimization.
\end{abstract}

Keywords: Metaheuristic algorithms, Continuous space optimization, Gravitational search algorithm (GSA), Black hole operator.

\section{Introduction}

Over the last decades, the attention to solve optimization problems with high-dimensional search space using metaheuristics has grown considerably. Metaheuristics consist of general approximate algorithms applicable to a wide range of optimization problems. The stochastic rules and inspiring by nature are of the prevailing features of metaheuristic algorithms. For instance, Evolutionary Algorithms, EAs, was conceptualized by chromosome and gene from Darwinian theory ${ }^{1}$; Particle Swarm Optimization, PSO, was designed from the behavior of flock of birds and fish schooling to find foods ${ }^{2,3}$; Simulated Annealing, SA, was constructed based on following the thermodynamic laws ${ }^{4}$; Ant Colony Optimization, ACO, mimics the behavior of ants to forage shortest path between their nest and a food source ${ }^{5}$; Big Bang BigCrunch, BB-BC, is inspired by the theories of the evolution of the universe named big bang and big crunch $^{6}$, etc. The metaheuristic algorithms have been widely used to solve many kinds of optimization problems such as industrial problems ${ }^{7}, 8,9,10$, filter modeling $^{11}$, networking ${ }^{12}$, modern physics $^{13}$, medicine $e^{14,15,16}$, economy ${ }^{17}$, robotics ${ }^{18,19,20}$ etc.

Although good performance of the metaheuristic search algorithms has been reported in many works by researchers, they are not able to solve all problems due to getting trapped in local optima. In metaheuristic search algorithms, exploration and exploitation are two effective abilities in progress of algorithms which should be balanced suitably to achieve good global and local search characteristics. The ability of finding new and better solutions in search space is exploration, and exploitation is the ability of finding the optima around good solutions. To enhance the performance of an 
algorithm, the trade-off between exploration and exploitation is necessary. In metaheuristic algorithms, there are some special operators which have their own abilities of exploration and exploitation. Therefore, metaheuristic optimization algorithms hybridize skillfully the operators together for a good trade-off between exploration and exploitation ${ }^{21}$. Thus, new operators are proposed or the available operators are redesigned in order to enhance particular abilities of metaheuristic algorithms for solving some problems. These algorithms have demonstrated the ability of solving hard computational problems within reasonable computation time. Some of such algorithms have been introduced in Refs. ${ }^{22-25}$.

Ref. $^{23}$ proposed a hybrid PSO that incorporates a mutation operator based on wavelet theory. In fact, the authors have used wavelet theory to enhance PSO in effectively exploring search space. In another work, Cheng et.al. ${ }^{24}$, hybridized PSO with GA operators i.e. mutation and crossover to find better solutions in a discrete space. The authors in Ref. ${ }^{25}$ proposed an improved version of ACO, called ant-weight strategy, which possesses a new strategy to update the pheromone trail and a mutation operation, to solve vehicle routing problem.

Gravitational Search Algorithm (GSA) is one of the relatively novel population based search algorithms introduced by Rashedi et.al. ${ }^{26,}{ }^{27}$. It is inspired by universal law of Newton's gravity. In GSA, the agents are considered as objects which are guided by the gravitational force. This force causes a global movement of all objects towards the objects with heavier masses ${ }^{26}$. This subject may lead the algorithm to a premature convergence for some situations. In other words, as this force absorbs the objects to each other, if premature convergence happens, there won't be any recovery for the algorithm. It means that after getting converged the algorithm loses its ability of exploration and then gets stagnated. Therefore, new operators should be appended to GSA in order to increase its flexibility for solving more complex problems. More details regarding the GSA stagnation have been reported by Sarafrazi et al. ${ }^{28}$.

In this order, Sarafrazi et al. ${ }^{28}$, proposed an operator called "disruption", originating from astrophysics, to improve the ability of GSA in exploration. In Ref. ${ }^{29}$ an improvement of GSA was introduced that gets the searching strategy of PSO and hybridizes it with GSA strategy. It means that the agents obey the law of gravity and receive the guidance from memory and social information.

In this paper, we propose a new operator named "Black Hole" to enhance the abilities of exploitation and exploration of GSA which develops our prior works on the multimodal problems ${ }^{30,31}$. The idea behind black hole operator is inspired by the astronomical phenomenon. This phenomenon has motivated researchers on various fields of studies. For example, in Ref. ${ }^{32}$, a new heuristic algorithm was designed for data clustering with the same name. The authors in Ref. ${ }^{33}$ proposed a model of data reorganization based on the black hole phenomenon. Also, a detector for video analysis is created inspired by this phenomenon ${ }^{34}$. It is declared that our work is different from other works that used the same name and is an extension of our previous works in this field ${ }^{30,31}$. Although the first version of the operator ${ }^{30,31}$ speeds up the movement of objects toward the best solution and it makes rapid convergence of the unimodal problems, it fails to overcome the stagnation situation in complicated multimodal problems. Therefore, in this paper we extend the operator by reformulating it to be able to effectively handle both unimodal and multimodal problems.

The remainder of this paper is organized as follows: in Section 2, GSA is reviewed. The concept of the black hole in nature is given in Section 3. In Section 4, the proposed black hole operator is explained. The performance of black hole operator is evaluated on standard benchmark functions and compared with GSA, Disruption GSA (DGSA), PSO and real-valued GA (RGA) and some well-known metaheuristics in Section 5. Finally, the paper is concluded in last Section.

\section{The Gravitational Search Algorithm}

The GSA is a swarm based metaheuristic algorithm inspired by the laws of gravity and motion. This algorithm ${ }^{26,27}$ causes agents to move in the search space to find optimum solution by using these natural laws. In other words, the information of fitness landscape is exchanged between the agents by Newton's gravity force, and then the agents move toward the promising areas, gradually.

In the GSA, the position of an agent (object) is shown by $X_{i}=\left(x_{i}^{1}, x_{i}^{2}, \ldots, x_{i}^{n}\right)$ where $n$ indicates the dimension. The positions are considered as candidate 
solutions. At first, the objects are distributed randomly in the search space. The mass value of each object is determined according to its fitness value ${ }^{27}$ :

$$
M_{i}=\frac{\operatorname{fit}_{i}(t)-\operatorname{worst}(t)}{\sum_{j=1}^{N} f i t_{j}(t)-\operatorname{worst}(t)},
$$

where $f i t_{i}(t)$ is the fitness value of object $i$ at iteration $t$; worst $(t)$ is the worst fitness value of the objects at time $t$, and $N$ is the number of objects or the size of swarm.

By the calculating the mass of objects, they can interact by each other by Eq. $2^{27}$ :

$F_{i}^{d}(t)=\sum_{j \neq i} r_{j} . G(t) \frac{M_{i}(t) \times M_{j}(t)}{R_{i j}(t)+\varepsilon} \cdot\left(x_{j}^{d}(t)-x_{i}^{d}(t)\right)$.

where $G(t)$ is the gravity constant; $\varepsilon$ is a very small value, and $R_{i j}(t)$ is the Euclidian distance between the two agents $i$ and $j$. Then, the acceleration of the agent is calculated by the second law of motion ${ }^{27}$ :

$$
a_{i}^{d}(t)=\frac{F_{i}^{d}(t)}{M_{i}(t)}
$$

Afterward, the Eqs. 4 and 5 are used to update the position of each agent. According to Eq. 4, the next velocity of the agent $i$ is calculated as a fraction of its current velocity added to its acceleration ${ }^{27}$.

$$
\begin{aligned}
& v_{i}^{d}(t+1)=r_{i} * v_{i}^{d}(t)+a_{i}^{d}(t), \\
& x_{i}^{d}(t+1)=x_{i}^{d}(t)+v_{i}^{d}(t+1) .
\end{aligned}
$$

where $r_{i}$ and $r_{j}$ are two uniform distributed random numbers in the interval $[0,1]$. Fig. 1 shows the pseudo code of the GSA.

1. Search space identification, $\mathrm{t}=0$;

2. Random initialization, $X_{i}(t)$;

For $i=1, \ldots, N$

3. Fitness evaluation of objects;

4. Update the parameters of $G$, worst and $M_{i}$;

For $i=1, \ldots, N$

5. Calculation of the force applied to each object;

6. Calculation of the acceleration and the velocity of each object;

7. Update the position of the objects by Eq. 5 to yield $X_{i}(t+1) ; t=t+1$

8. Repeat steps 3 to 7 until the stopping criterion is reached; 9. end

Fig. 1. The pseudo code for standard GSA (SGSA).

\section{The Nature of Black Holes}

As we know, the black hole is known as one of the wonderful phenomena in the world. This is amazing because it has a so extremely strong gravity that even light could not escape from it. In this section, we review some features of black holes and will inspire some of them in our work. Now, a star is visible to us because light escapes from its surface. As long ago as the late 1700's, the British physicist John Michell and the French mathematician and physicist Pierre Laplace speculated (independently) on the possibility that stars might exist whose escape velocity was larger than the speed of light. Michell and Laplace both understood that if nature was able to make a star more compact than the Sun, but with the same mass, then it would have a larger escape velocity ${ }^{36}$. In 1939, American physicists, J. Robert Oppenheimer and Hartland Snyder described that a sufficient heavy star will collapse when all the thermonuclear sources of energy are exhausted ${ }^{37}$. To escape an object from the gravity of a star, the following condition by conservation of energy must be satisfied:

$$
\frac{1}{2} m v^{2}>\frac{G m M}{R}
$$

where $\mathrm{m}$ is the mass of object; $M$ is the mass of star; $v$ is the velocity of object; $G$ is gravity constant and $R$ is the distance of object from center of star. Actually, the kinetic energy of the object must be more than the gravitational energy of star. We assume that the object is photon and it possesses velocity of light i.e. $(v \rightarrow c)$. Therefore, it is a region around of star where prevents the object from escaping. According to Eq. 6, the region is calculated as follows:

$$
R_{S}=\frac{2 G M}{C^{2}},
$$

where $c$ is the velocity of light and $R_{S}$ is called "Schwarzschild radius" or "event horizon". Since nothing can escape from this horizon $\left(r=R_{S}\right)$, not even light, the star that has collapsed down within the Schwarzschild radius is called a black hole. Although the interior of a black hole, inside the event horizon, is a region that is forever hidden from us on the outside, its properties may still be calculated. At the center of a black hole there is a singularity point, which has zero volume and infinite density; i.e. all of the black hole's mass is located there ${ }^{36}$. Near a rotating black hole, there is a nonspherical region outside the event horizon called the Ergosphere where any object must move spirally in the same direction that the black hole rotates. The important regions of black hole are shown in Fig. $2^{36}$.

The movement of the object fallen in the black hole can be explained as follows ${ }^{37}$ : 


$$
\frac{d t}{d r}=-\frac{2 M}{r-2 M} .
$$

Therefore, we have by integration:

$$
t=-2 M \ln \left(\frac{r}{2 M}-1\right)+\text { const }
$$

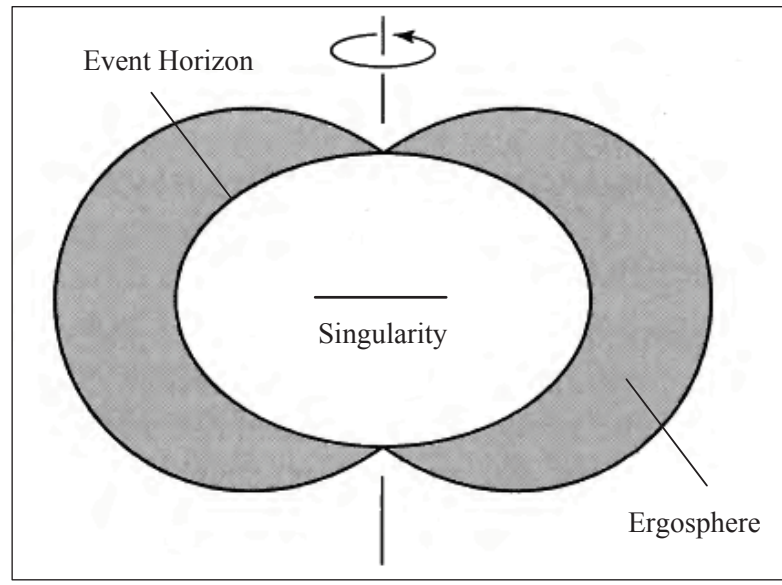

Fig. 2. The structure of rotating black holes.

where $t$ is time and $r$ is distance of the object to black hole. Thus, we have:

$$
r=2 M\left(1+\exp \left(-\frac{t-\text { const }}{2 M}\right)\right)
$$

The surface area of the event horizon of a black hole has the remarkable property that it always increases when additional matter falls into the hole. Moreover, if two black holes collide and merge to form a single hole, the area of the new horizon is greater than the sum of the areas of the colliding holes. It is also mentioned as one of the other characteristics of black holes that an object falling into a black hole experiences very strong forces known as tidal forces. In fact, the strong gravitational force effects tear the object to pieces, and the object is fragmented ${ }^{38}$.

\section{Simulation of the Black Hole as a Gravitational Operator}

We design an operator based on some characteristics of the black hole phenomenon. The proposed operator is inserted in standard GSA as a new operator to solve different problems. To simulate the black hole phenomenon, it is assumed that the best solution, is as star that becomes the black hole and attracts other solutions. The black hole operator (BH) affects the heavy and light objects in two different ways. Each swarm's member is categorized into heavy or light object according to its mass. The updating position of each object depends on the distance of the object from the black hole and the Schwarzschild radius of the black hole. To balance the exploration and exploitation, we define two Schwarzschild radiuses for two categories of heavy and light objects $\left(R_{S}, R_{S}^{\prime}\right)$, respectively as follows:

$$
\begin{aligned}
& R_{s}=G M v^{2} / t, \\
& R_{s}^{\prime}=M \cdot \log (t),
\end{aligned}
$$

where $G$ is gravity constant; $R_{S}$ and $R_{S}^{\prime}$ are Schwarzschild radiuses for a black hole when encountering heavy and light masses, respectively, $M$ is the mass of black hole; $t$ indicates the number of algorithm's iterations and $v$ is the velocity of the object which is determined as minimum velocity value for each object.

In fact, the first category, which contains the heavy objects, causes the agents to see around the black hole and push them to a better solution. The second category is embedded to more explore the search space by changing the position of light objects (the objects with low fitness).

The proposed black hole operator chooses the most massive object as the black hole at the first. Then, the reminder objects of the swarm are divided into two categories based on their masses (fitness values). On the other hand, the Schwarzschild radiuses of the black hole are determined for affecting on heavy and light objects according to Eqs. 11 and 12, respectively. Finally, the updating positions of objects are done as follows:

- The position updating of heavy objects:

The Schwarzschild radius of the black hole for heavy objects $\left(R_{S}\right)$ is determined by Eq. 11, and the distance of each heavy object from the black hole is measured $(r)$. For those of heavy objects that satisfied $r<R_{s}$, the position is changed as follows:

$x_{i}^{d}(t+1)=x_{i}^{d}(t)+\operatorname{rand} .\left(x_{B H}^{d}(t)-x_{i}^{d}(t)\right)$,

where $x_{B H}^{d}$ is the position of black hole in dimension $d$ and rand is an uniformly distributed random number in the interval $[0,1]$.

- The position updating of light objects:

The Schwarzschild radius of the black hole for light objects $\left(R_{S}^{\prime}\right)$ is determined by Eq. 12, and the distance of each light object from the black hole is measured 
$\left(r^{\prime}\right)$. The position of a light agent is changed as below if it is satisfied $r^{\prime}<R_{S}^{\prime}$ :

$$
x_{i}^{d}(t+1)=\operatorname{rand} .\left(x_{i}^{d}(t) \cdot\left(r^{\prime} / R_{s}^{\prime}\right)\right) .
$$

1. Search space identification, $\mathrm{t}=0$;

2. Random initialization, $X_{i}(t)$;

For $i=1, \ldots, N$

3. Fitness evaluation of objects;

4. Update the parameters of $G$, worst and $M$;

For $i=1, \ldots, N$

5. Calculation of the force applied to each object;

6. Calculation of the acceleration and the velocity of each object;

7. Appling Eq. 5 to obtain position of agents;

8. Update the position of the agents by Eqs. 13 and 14 to yield $X_{i}(t+1) ; t=t+1$;

9. Repeat steps 3 to 8 until the stopping criterion is reached; 10. end

Fig. 3. The Pseudo code for the proposed algorithm.

\section{Experimental Results}

Two sets of benchmark functions are used to evaluate the performance of our proposed algorithm. The first set includes 23 benchmark functions that are divided into three categories: unimodal functions, multimodal high dimensional functions, and multimodal low dimensional functions. In the first category, the convergence rate is more important than final results. Functions $F_{1}$ to $F_{7}$ are unimodal functions and they are defined in Table 1 . The multimodal high dimensional functions have many local optima and the final results are more important. These functions are shown in Table 2. The last category of the benchmark functions that having no many local optima, are defined in Table 3. In these tables, $n$ is the number of dimension of the function; $f_{\text {opt }}$ is the minimum value of the function, and $S \subseteq R^{n}$ defines the search space. A detail description of these functions can be found in Refs. ${ }^{26,27}$ and ${ }^{39}$. The CEC 2005 benchmark functions ${ }^{42}$ are used as second set of benchmark functions to evaluate the performance of the proposed algorithm. These functions are explained in subsection 5.3.

The performance of the proposed approach (BHGSA), is compared with some well-known optimization algorithms such as Particle Swarm Optimization, $\mathrm{PSO}^{3}$ Real Genetic Algorithm, RGA, ${ }^{40}$ Gravitational Search Algorithm, GSA, ${ }^{26}$ and Disruption Gravitational Search Algorithm, DGSA. ${ }^{28}$ The details of comparative algorithms are as follow:

In PSO, $x_{i}^{d}$ and $v_{i}^{d}$ are calculated as follow:

$$
\begin{gathered}
x_{i}^{d}(t+1)=x_{i}^{d}(t)+v_{i}^{d}(t+1), \\
v_{i}^{d}(t+1)=w(t) v_{i}^{d}(t)+c_{1} r_{i 1}\left(\text { pbest }_{i}^{d}-x_{i}^{d}\right)+ \\
c_{2} r_{i 2}\left(\text { gbest }_{i}^{d}-x_{i}^{d}\right) .
\end{gathered}
$$

where $r_{i 1}$ and $r_{i 2}$ are two uniformly distributed random numbers in the range $[0,1], c_{1}$ and $c_{2}$ are two positive constant which $c_{1}=c_{2}=2$ and inertia factor $(w)$ is decreased linearly from 0.9 to $0.2 . x_{i}^{d}$ and $v_{i}^{d}$ represent position and velocity of $i$-th particle in $d$-th dimension, respectively. Also, pbest $_{i}=\left(\right.$ pbest $_{i}^{1}$, pbest $_{i}^{2}, \ldots$, pbest $\left._{i}^{n}\right)$ and gbest $_{i}=$ $\left(\right.$ gbest $_{i}^{1}$, gbest $_{i}^{2}, \ldots$, gbest $\left._{i}^{n}\right)$ respectively represent the best previous position of $i$-th particle and the best previous position among all particles in the population. In RGA, arithmetic crossover, Gaussian mutation and roulette wheel selection were used, as described in Ref. $^{40}$ and the crossover and mutation probabilities were set to 0.3 and 0.1 , respectively.

In all forms of GSAs (GSA, DSGA and BH-GSA), $G$ is defined as Eq. 17 , where $G_{0}$ is set to $100 ; t_{\text {Max }}$ is the total number of iterations (the total age of system), and $\alpha$ is set to $20,{ }^{26}$ :

$$
G=G_{0} \exp \left(-\alpha \frac{t}{t_{\operatorname{Max}}}\right) .
$$

Finally, in DGSA to implement the Disruption operator the following equations are used as ${ }^{28}$

$$
\begin{gathered}
\frac{R_{i, j}}{R_{i, \text { best }}}<C, \\
x_{i} \text { (new) }=x_{i}(\text { old }) . D, \\
D=\left\{\begin{array}{l}
R_{i, j} \cdot U(-0.5,0.5), \text { if } R_{i, \text { best }} \geq 1 \\
(1+\rho . U(-0.5,0.5), \text { otherwise }
\end{array}\right.
\end{gathered}
$$

where $R_{i, j}$ and $R_{i, b e s t}$ are Euclidean distances between objects $i$ and $j$ and between object $i$ and the best solution, respectively. In Eq. 20, $\rho$ is a small constant which is set to $10^{-16}$, also $U(-0.5,0.5)$ returns a uniformly distributed random number in the interval $[-0.5,0.5]$. Also $C$ is decreased linearly by time as below:

$$
C=\theta\left(1-\frac{t}{t_{\operatorname{Max}}}\right),
$$

where $\theta$ is set to 100 , and $t_{\text {Max }}$ is the total number of iterations.

The Schwarzschild radiuses are defined using Eqs. 11 and 12, for the BH-GSA. Also the number of light objects are set to $N / 5$, and others are considered as heavy objects. For all functions, the results are 
averaged over 30 independent runs. The average bestso-far solutions, median of the best solutions, best of the best solutions and standard deviation of the best solutions in the last iteration of 30 runs are reported for all functions in Tables 4-6. For all algorithms, the population size is set to $50(N=50)$. The dimension is set to $30(n=30)$, and the maximum iteration $\left(t_{\text {Max }}\right)$ is 1000 for functions of Tables 1 and 2; the maximum iteration is set to 500 for the functions of Table 3 .

Table 1. Unimodal test functions.

\begin{tabular}{lcl}
\hline Test functions & $\boldsymbol{S}$ & $\boldsymbol{f}_{\text {opt }}$ \\
\hline$F_{1}(x)=\sum_{i=1}^{n} x_{i}^{2}$ & {$[-100,100]^{\mathrm{n}}$} & 0 \\
$F_{2}(x)=\sum_{i=1}^{n}\left|x_{i}\right|+\prod_{i=1}^{n}\left|x_{i}\right|$ & {$[-10,10]^{\mathrm{n}}$} & 0 \\
$F_{3}(x)=\sum_{i=1}^{n}\left(\sum_{j=1}^{i} x_{j}\right)^{2}$ & {$[-100,100]^{\mathrm{n}}$} & 0 \\
$F_{4}(x)=\max _{i}\left\{\left|x_{i}\right|, 1 \leq i \leq n\right\}$ & {$[-100,100]^{\mathrm{n}}$} & 0 \\
$F_{5}(x)=\sum_{i=1}^{n-1}\left[100\left(x_{i+1}-x_{i}{ }^{2}\right)^{2}+\left(x_{i}-1\right)^{2}\right]$ & {$[-30,30]^{\mathrm{n}}$} & 0 \\
$F_{6}(x)=\sum_{i=1}^{n}\left(\left[x_{i}+0.5\right]\right)^{2}$ & {$[-100,100]^{\mathrm{n}}$} & 0 \\
$F_{7}(x)=\sum_{i=1}^{n} i x_{i}^{4}+$ random $[0,1)$ & {$[-1.28,1.28]^{\mathrm{n}}$} & 0 \\
\hline
\end{tabular}

\subsection{A Comparison with other algorithms}

In Tables 4-6, the comparison results for competing algorithms (RGA, PSO, SGSA, DGSA, and BH-GSA) are reported. In these tables, the values that are less than $10^{-15}$ are set to zero. The obtained results for unimodal functions, given in Table 4, show that the BH-GSA provides acceptable results for most benchmark functions. In more details, the performance of the proposed algorithm is similar to DGSA and overall better than other competing algorithms.

In $F_{6}$, BH-GSA provides better results than DGSA whereas in $F_{5}$, DGSA performs better than BH-GSA. However, the BH-GSA and DGSA have the same performance in $F_{7}$ and they are better than SGSA. In all unimodal functions used in this section, the performance of PSO and RGA is worse than GSAs.

As early mentioned, the multimodal functions have many local optima and they are useful to evaluate the ability of search algorithm for escaping from poor local optima. The number of local optima in these functions will increase exponentially by increase of dimension. The dimension for these functions is set to $30(n=30)$.

Based on the results of Table 5, the proposed algorithm has powerful ability to explore the search space for optimum solution. The performance of BHGSA in finding optima for functions of Table 2 is superior than others, except for $F_{8}$ and $F_{12}$. In $F_{8}$, DGSA and RGA perform better than BH-GSA, SGSA and PSO. The good performance of our algorithm can be observed in $F_{9}, F_{10}, F_{11}$ and $F_{13}$. However, the performance of BH-GSA and DGSA is the same for $F_{9}$, $F_{10}$, and $F_{11}$. In case of $F_{13}$, BH-GSA, SGSA and PSO are more robust in exploiting than DGSA and RGA.

The multimodal low-dimensional functions are employed to investigate both exploration and exploitation abilities of the algorithms. Table 6 reports the obtained results of competing algorithms on the benchmark functions of Table 3. It shows that BH-GSA provide acceptable results for all functions and could find the optimal solution for most functions.

\subsection{Validation with nonparametric tests}

To evaluate the significance of the proposed algorithm, we employed Wilcoxon signed ranks nonparametric test for unimodal and multimodal benchmark functions, separately. At first, we used a pairwise test which compares the performance of two algorithms when applied to a common set of problems. The Wilcoxon signed ranks determines the sum of ranks $\left(R^{+}\right)$for the problems in which the first algorithm outperforms the second, and the sum of ranks for the opposite $\left(R^{-}\right)$. Both parameters are calculated in order to highlight the difference between the performance scores of two competing algorithms. The results of Wilcoxon signed ranks for competing algorithms in the cases of unimodal and multimodal benchmark functions are reported in Tables 7 and 8, respectively. The second nonparametric test that is employed here, is a multiple comparison called Friedman test. It is a nonparametric test to detect significant differences between the behavior of two or more algorithms. A detailed description of these tests is available in Ref. ${ }^{41}$. In all cases of the experiments, $\alpha$ is set to 0.05 as the level of confidences. The results obtained by Friedman test are reported in Tables 9-12. The obtained results confirmed the effectiveness of the proposed operator to make a more powerful GSA.

According to the results of Tables 7 and 9, the performance of the proposed algorithm is approximately equal to the performance DGSA for unimodal functions of set 1 . Table 7 reveals that DGSA is slightely better than BH-GSA based on the Wilcoxon signed ranks and Table 9 shows that BH-GSA outperforms the DGSA according to Friedman test.

Tables 8 and 11 confirm that BH-GSA outperforms all competing algorithms based on Wilcoxon signed ranks and Friedman tests for multimodal functions of set 1 . 
Table 2. Multimodal test functions.

\begin{tabular}{lcc}
\hline Test functions & $\boldsymbol{S}$ & $\boldsymbol{f}_{\text {opt }}$ \\
\hline$F_{8}(x)=\sum_{i=1}^{n}-x_{i} \sin \left(\sqrt{\left|x_{i}\right|}\right)$ & {$[-500,500]^{\mathrm{n}}$} & $-418.9829 \times n$ \\
$F_{9}(x)=\sum_{i=1}^{n}\left[x_{i}^{2}-10 \cos \left(2 \pi x_{i}\right)+10\right]$ & {$[-5.12,5.12]^{\mathrm{n}}$} & 0 \\
$F_{10}(x)=-20 \exp \left(-0.2 \sqrt{\frac{1}{n}} \sum_{i=1}^{n} x_{i}^{2}\right)-\exp \left(\frac{1}{n} \sum_{i=1}^{n} \cos \left(2 \pi x_{i}\right)\right)+20+e$ & {$[-32,32]^{\mathrm{n}}$} & 0 \\
$F_{11}(x)=\frac{1}{4000} \sum_{i=1}^{n} x_{i}^{2}-\prod_{i=1}^{n} \cos \left(\frac{x_{i}}{\sqrt{i}}\right)+1$ & {$[-600,600]^{\mathrm{n}}$} & 0 \\
$F_{12}(x)=\frac{\pi}{n}\left\{10 \sin ^{2}\left(\pi y_{1}\right)+\sum_{i=1}^{m-1}\left(y_{i}-1\right)^{2}\left[1+10 \sin ^{2}\left(\pi y_{i+1}\right)\right]+\left(y_{i}-1\right)^{2}\right\}$ & {$[-50,50]^{\mathrm{n}}$} & 0 \\
$\quad+\sum_{i=1}^{n} u\left(x_{i}, 10,100,4\right)$ & \\
$y_{i}=1+\frac{x_{i}+1}{4}, \mathrm{u}\left(x_{i}, a, k, m\right)=\left\{\begin{array}{c}k\left(x_{i}-a\right)^{m}-a<x_{i}<a \\
0\left(-x_{i}-a\right)^{m} \quad x_{i}<a\end{array}\right.$ & {$[-50,50]^{\mathrm{n}}$} & \\
$F_{13}(x)=0.1\left\{\sin ^{3}\left(3 \pi x_{i}\right)+\sum_{i=1}^{n}\left(x_{i}-1\right)^{2}\left[1+\sin ^{2}\left(3 \pi x_{i}+1\right)\right]\right.$ & \\
$\left.\quad+\left(x_{n}-1\right)^{2}\left[1+\sin ^{2}\left(2 \pi x_{n}\right)\right]\right\}+\sum_{i=1}^{n} u\left(x_{i}, 5,100,4\right)$ & 0
\end{tabular}

Table 3. Multimodal test functions with fix dimension.

\begin{tabular}{lll}
\hline Test functions & \multicolumn{1}{c}{$\boldsymbol{S}$} & $\boldsymbol{f}_{\text {opt }}$ \\
\hline$F_{14}(x)=\left(\frac{1}{500}+\sum_{j=1}^{25} \frac{1}{j+\sum_{i=1}^{2}\left(x_{i}-a_{i j}\right)^{6}}\right)^{-1}$ & {$[-65.53,65.53]^{2}$} & 1 \\
$F_{15}(x)=\sum_{i=1}^{11}\left[a_{i}-\frac{x_{1}\left(b_{i}^{2}+b_{i} x_{2}\right.}{b_{i}^{2}+b_{i} x_{3}+x_{4}}\right]^{2}$ & {$[-5,5]^{4}$} & 0.00030 \\
$F_{16}(x)=4 x_{1}^{2}-2.1 x_{1}^{4}+\frac{1}{3} x_{1}^{6}+x_{1} x_{2}-4 x_{2}^{2}+4 x_{2}^{4}$ & {$[-5,5]^{2}$} & -1.0316 \\
$F_{17}(x)=\left(x_{2}-\frac{5.1}{4 \pi^{2}} x_{1}^{2}+\frac{5}{\pi} x_{1}-6\right)^{2}+10\left(1-\frac{1}{8 \pi}\right) \cos x_{1}+10$ & {$[-5,10] \times[0,15]$} & 0.398 \\
$F_{18}(x)=\left[1+\left(x_{1}+x_{2}+1\right)^{2}\left(19-14 x_{1}+3 x_{1}^{2}-14 x_{2}+\sum_{i=1}^{n} u\left(x_{i}, 10,100,4\right)\right.\right.$ & {$[-5,5]^{2}$} & 3 \\
$\left.\left.+6 x_{1} x_{2}+3 x_{2}^{2}\right)\right] \times\left[30+\left(2 x_{1}-3 x_{2}\right)^{2} \times\left(18-32 x_{1}+12 x_{1}^{2}+48 x_{2}-36 x_{1} x_{2}+27 x_{2}^{2}\right)\right]$ & {$[0,1]^{3}$} & $-3,1]^{6}$ \\
$F_{19}(x)=-\sum_{i=1}^{4} c_{i} \exp \left(-\sum_{i=1}^{3} a_{i j}\left(x_{j}-p_{i j}\right)^{2}\right)$ & {$[0,10]^{4}$} & -3.86 \\
$F_{20}(x)=-\sum_{i=1}^{4} c_{i} \exp \left(-\sum_{i=1}^{6} a_{i j}\left(x_{j}-p_{i j}\right)^{2}\right)$ & {$[0,10]^{4}$} & -3.32 \\
$F_{21}(x)=-\sum_{i=1}^{5}\left[\left(X-a_{i}\right)\left(X-a_{i}\right)^{T}+c_{i}\right]^{-1}$ & -10.1532 \\
$F_{22}(x)=-\sum_{i=1}^{7}\left[\left(X-a_{i}\right)\left(X-a_{i}\right)^{T}+c_{i}\right]^{-1}$ & {$[0,10]^{4}$} & -10.4029 \\
$F_{23}(x)=-\sum_{i=1}^{10}\left[\left(X-a_{i}\right)\left(X-a_{i}\right)^{T}+c_{i}\right]^{-1}$ & -10.5363 \\
\hline
\end{tabular}

\subsection{The Experimental Results of BH-GSA on the CEC 2005 benchmark functions}

The proposed algorithm is also tested on 25 standard benchmark functions of CEC 2005. These functions are summarized in Table 13 and are available in Ref. ${ }^{42}$. Functions 1-5 are unimodal and 6-12 are multimodal. Functions 13-25 are hybrid composition functions. Table 21 contains the result error values for these functions after $1 \mathrm{e}+3,1 \mathrm{e}+4$ and $1 \mathrm{e}+5$ fitness evaluations (FEs) with dimension $10(\mathrm{n}=10)$. For each function, 25 independent runs are executed and minimum $\left(1^{\text {th }}\right),\left(7^{\text {th }}\right)$ median $\left(13^{\text {th }}\right),\left(19^{\text {th }}\right)$, and maximum values $\left(25^{\text {th }}\right)$, as well as mean values and standard deviation are given in this table. The parameters of BH-GSA are set as the prior experiments.

The performance of BH-GSA is compared with some other metaheuristic search algorithms including Evolutionary Strategy (ES) ${ }^{43}$, Parameter-less Evolution
Strategy (PLES) ${ }^{43}$, DGSA and Quantum Particle Swarm Optimization (QPSO) ${ }^{44}$ in Table 14.

In more details, for unimodal functions, BH-GSA performed well for functions $F_{1}, F_{2}$ and $F_{4}$. However, this algorithm could not achieve optimal solutions in $F_{3}$ and $F_{5}$. For multimodal functions, the proposed algorithm have better performance than other algorithms for $F_{6}, F_{8}, F_{10} F_{11}, F_{12}$ and $F_{15}-F_{24}$. However, for some functions such as $F_{7}$ and $F_{25} \mathrm{BH}-$ GSA does not have a good performance.

The function $F_{7}$ is a non-separable and scalable multi-modal that these properties make it hard to analysis. The ES and PLES outperform BH-GSA, DGSA, and QPSO in this function. For the fuction $F_{8}$, the proposed algorithm has acceptable performance comparing with other algorithms. The global optimum of this fuction is located at the boundary as shown in Fig. 4. The result of $F_{9}$ shows that although QPSO and BH-GSA have approximately good performance, but QPSO has found better solution than our algorithm. 
However, according to Table 14 the BH-GSA significantly preforms better than other competing algorithms for $F_{10}$ and $F_{11}$. These functions are shifted, rotated, non-separable, and scalable multi-modal problems. A huge number of local optima for $F_{11}$ are depicted in Fig. 5. Also, this good trend of our algorithm is observable in $F_{8}, F_{12}, F_{16}$, and $F_{17}$. In case of $F_{12}$, our work has achieved a better solution than other algorithms. However, QPSO performs better than others in the results of $F_{13}$ and $F_{14}$, BH-GSA also tries to find good solutions in these functions and its performance is near to QPSO's results. These functions are shifted, non-separable, and scalable multi-modal problems. In addition, BH-GSA has better results than competing algorithms for $F_{15}$ and $F_{18}-F_{23}$, but it still could not find the optima. These functions are difficult to analysis. For example, This subject can be seen for the function $F_{19}$ in Fig. 6.

In the function $F_{25}$, all algorithms have similar conditions, while our algorithm provides better result than others in $F_{24}$. These functions are both nonseparable multi-modal and scalable with a huge number of local optima. The 2-dimension fitness landscape of $F_{24}$ is given in Fig. 7 .

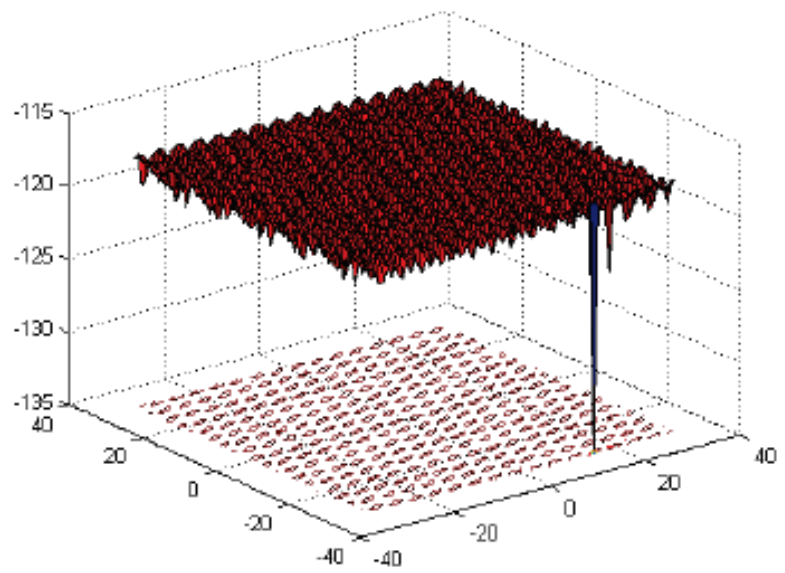

Fig. 4. The 2-dimensional fitness landscape of $F_{8}$.

The results of nonparametric tests for functions of set 2 are reported in Tables 15-20. According to these tables, BH-GSA outperforms all competing algorithms in multimodal functions of CEC 2005. It should be mentioned that QPSO performs better than our algorithm in unimodal functions and the BH-GSA could get the second rank for unimodal functions of this set. These results show that BH-GSA is significantly better than DGSA in all functions of CEC 2005. Also the BH-GSA performs better than ES and PLES for all functions except for $F_{7}, F_{14}$, and $F_{25}$.

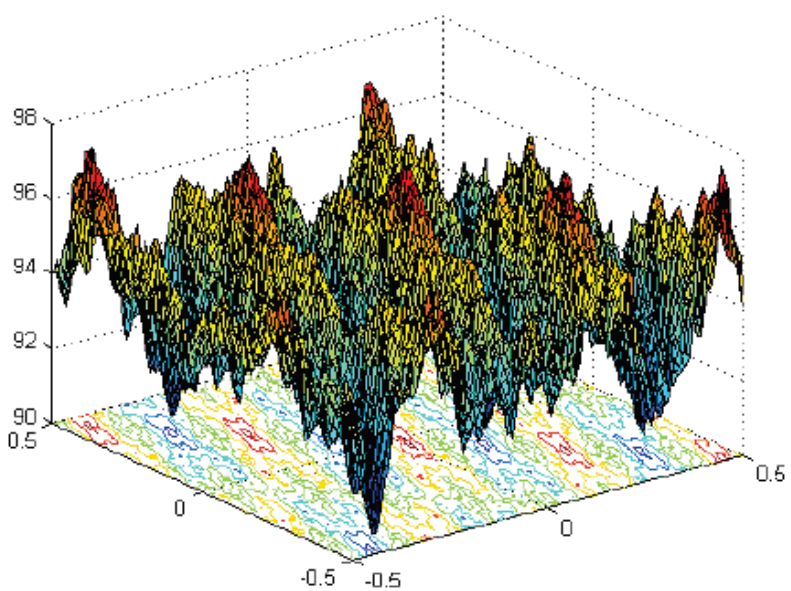

Fig. 5. The 2-dimensional fitness landscape of $F_{11}$.

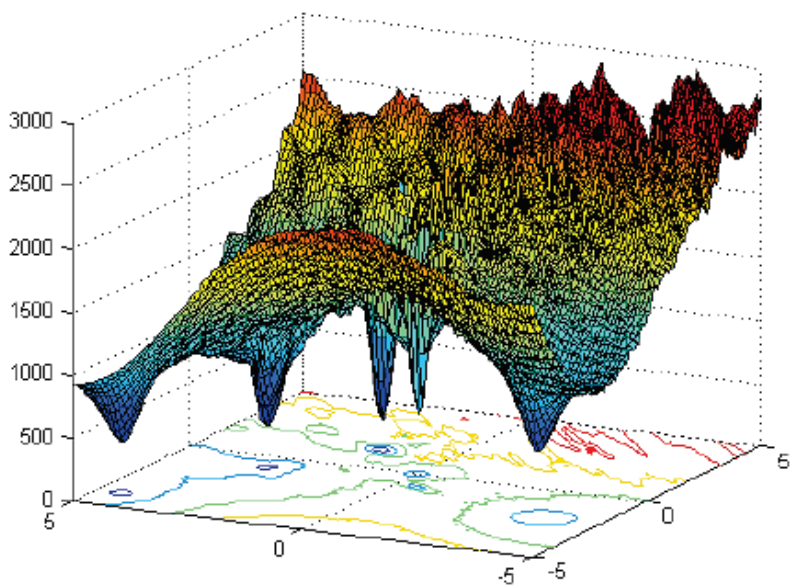

Fig. 6. The 2-dimensional fitness landscape of $F_{19}$.

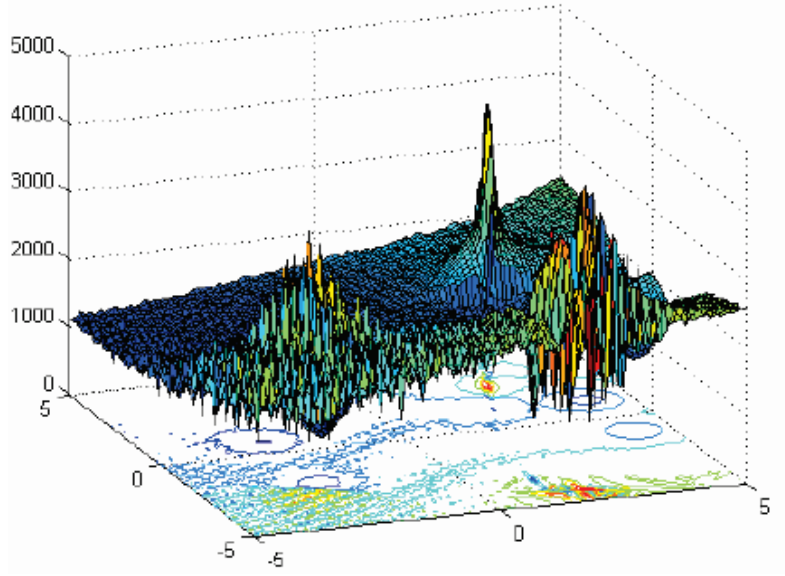

Fig. 7. The 2-dimensional fitness landscape of $F_{24}$. 
Table 4. Minimization result of benchmark functions in Table 1 with $n=30$ and $t_{\text {Max }}=1000$.

\begin{tabular}{|c|c|c|c|c|c|c|}
\hline Functions & & RGA & $\mathrm{PSO}$ & SGSA & DGSA & BH-GSA \\
\hline \multirow{4}{*}{$F_{1}$} & Average best-so-far & 23.1310 & 0.0017 & 0 & 0 & 0 \\
\hline & Median best-so-far & 21.8710 & 0.0012 & 0 & 0 & 0 \\
\hline & Best best-so-far & 9.6389 & $1.06 \times 10^{-4}$ & 0 & 0 & 0 \\
\hline & Std best-so-far & 12.1693 & 0.0021 & 0 & 0 & 0 \\
\hline \multirow{4}{*}{$F_{2}$} & Average best-so-far & 1.0736 & 2.0014 & $2.25 \times 10^{-8}$ & 0 & 0 \\
\hline & Median best-so-far & 1.1371 & 0.0019 & $3.16 \times 10^{-8}$ & 0 & 0 \\
\hline & Best best-so-far & 0.6531 & $7.42 \times 10^{-4}$ & $1.02 \times 10^{-8}$ & 0 & 0 \\
\hline & Std best-so-far & 0.2676 & 4.2112 & $3.46 \times 10^{-9}$ & 0 & 0 \\
\hline \multirow{4}{*}{$F_{3}$} & Average best-so-far & 561.69 & 411.71 & 238.18 & 0 & 0 \\
\hline & Median best-so-far & 569.19 & 226.27 & 221.59 & 0 & 0 \\
\hline & Best best-so-far & 395.37 & 138.93 & 97.66 & 0 & 0 \\
\hline & Std best-so-far & 125.83 & 322.42 & 101.11 & 0 & 0 \\
\hline \multirow{4}{*}{$F_{4}$} & Average best-so-far & 11.7791 & 8.1594 & $4.68 \times 10^{-9}$ & 0 & 0 \\
\hline & Median best-so-far & 11.8987 & 7.4562 & $2.79 \times 10^{-9}$ & 0 & 0 \\
\hline & Best best-so-far & 9.3587 & 5.5419 & $5.06 \times 10^{-9}$ & 0 & 0 \\
\hline & Std best-so-far & 1.5821 & 2.4127 & $8.01 \times 10^{-10}$ & 0 & 0 \\
\hline \multirow{4}{*}{$F_{5}$} & Average best-so-far & $1.16 \times 10^{+3}$ & $3.64 \times 10^{+4}$ & 29.7591 & 0.6714 & 26.6901 \\
\hline & Median best-so-far & $1.05 \times 10^{+3}$ & $4.13 \times 10^{+3}$ & 26.0653 & 0.3104 & 26.5513 \\
\hline & Best best-so-far & 544.9892 & 82.2979 & 25.7613 & $1.71 \times 10^{-5}$ & 26.2003 \\
\hline & Std best-so-far & 548.0063 & $4.51 \times 10^{+4}$ & 18.8949 & 1.4135 & 0.5468 \\
\hline \multirow{4}{*}{$F_{6}$} & Average best-so-far & 24.0129 & 0.0010 & 0 & 0.0055 & 0 \\
\hline & Median best-so-far & 24.5589 & $5.49 \times 10^{-4}$ & 0 & 0.0031 & 0 \\
\hline & Best best-so-far & 4.0397 & $7.10 \times 10^{-5}$ & 0 & $6.09 \times 10^{-8}$ & 0 \\
\hline & Std best-so-far & 10.1753 & 0.0012 & 0 & 0.0024 & 0 \\
\hline \multirow{4}{*}{$F_{7}$} & Average best-so-far & 0.0675 & 0.0433 & 0.0169 & $2.65 \times 10^{-5}$ & $2.37 \times 10^{-5}$ \\
\hline & Median best-so-far & 0.0635 & 0.0432 & 0.0145 & $1.81 \times 10^{-5}$ & $2.59 \times 10^{-5}$ \\
\hline & Best best-so-far & 0.0323 & 0.0332 & 0.0012 & $3.28 \times 10^{-7}$ & $9.53 \times 10^{-6}$ \\
\hline & Std best-so-far & 0.0246 & 0.0069 & 0.0104 & $2.47 \times 10^{-5}$ & $1.98 \times 10^{-5}$ \\
\hline
\end{tabular}

\section{Conclusion}

The interest in optimization by metaheuristics has grown recently in many fields of sciences to solve different problems. The most of these algorithms are inspired by nature. One of the relatively novel metaheuristics is GSA which is established by concept of motion and law of gravity. In this paper, a new operator inspired by one of the astrophysical phenomena, called black hole, is proposed. We studied some physical features of this phenomenon and simulated it as an search operator to improve the performance of GSA. This algorithm is implemented simply and not only has the powerful ability to explore, but it is capable in exploiting as well. To evaluate the proposed algorithm, two sets of standard benchmark functions are employed and the results are compared with some well-known algorithms. Also, the results have been validated by two nonparametric tests. The results obtained confirm the significant performance of the proposed operator. In the future, the proposed algorithm is used to solve different optimization problems in many fields of research.

\section{Acknowledgements}

The authors would like to thank Mrs Z. Aref \& Z. Karimi Soflu for providing valuable comments. 
Table 5. Minimization result of benchmark functions in Table 2 with $n=30$, and $t_{\text {Max }}=1000$.

\begin{tabular}{|c|c|c|c|c|c|c|}
\hline Functions & & RGA & PSO & SGSA & DGSA & BH-GSA \\
\hline \multirow{4}{*}{$F_{8}$} & Average best-so-far & $-1.2483 \times 10^{+4}$ & $-9.88 \times 10^{+3}$ & $-2.82 \times 10^{+3}$ & $-1.2569 \times 10^{+4}$ & $-2.9901 \times 10^{+3}$ \\
\hline & Median best-so-far & $-1.2496 \times 10^{+4}$ & $-2.8 \times 10^{+3}$ & $-2.83 \times 10^{+3}$ & $-1.2569 \times 10^{+4}$ & $-3.0269 \times 10^{+3}$ \\
\hline & Best best-so-far & $-1.2523 \times 10^{+4}$ & $-1.06 \times 10^{+4}$ & $-3.67 \times 10^{+3}$ & $-1.2569 \times 10^{+4}$ & $-3.6369 \times 10^{+3}$ \\
\hline & Std best-so-far & 53.2640 & 512.22 & 404.55 & 0.0010 & 322.8889 \\
\hline \multirow{4}{*}{$F_{9}$} & Average best-so-far & 5.9020 & 55.1429 & 15.52 & 0 & 0 \\
\hline & Median best-so-far & 5.7165 & 55.6035 & 15.91 & 0 & 0 \\
\hline & Best best-so-far & 3.7858 & 35.3898 & 8.95 & 0 & 0 \\
\hline & Std best-so-far & 1.1710 & 15.4611 & 3.60 & 0 & 0 \\
\hline \multirow{4}{*}{$F_{10}$} & Average best-so-far & 2.1395 & 0.0090 & $3.31 \times 10^{-9}$ & 0 & 0 \\
\hline & Median best-so-far & 2.1680 & 0.0066 & $3.34 \times 10^{-9}$ & 0 & 0 \\
\hline & Best best-so-far & 1.3778 & 0.0031 & $6.29 \times 10^{-9}$ & 0 & 0 \\
\hline & Std best-so-far & 0.4014 & 0.0076 & $5.62 \times 10^{-10}$ & 0 & 0 \\
\hline \multirow{4}{*}{$F_{11}$} & Average best-so-far & 1.1676 & 0.0101 & 3.99 & 0 & 0 \\
\hline & Median best-so-far & 1.1399 & 0.0081 & 3.89 & 0 & 0 \\
\hline & Best best-so-far & 1.0469 & $5.14 \times 10^{-4}$ & 1.24 & 0 & 0 \\
\hline & Std best-so-far & 0.0801 & 0.0093 & 1.42 & 0 & 0 \\
\hline \multirow{4}{*}{$F_{12}$} & Average best-so-far & 0.0510 & 0.2926 & 0.0524 & $4.12 \times 10^{-4}$ & 0.0041 \\
\hline & Median best-so-far & 0.0399 & 0.1140 & $5.42 \times 10^{-19}$ & $1.15 \times 10^{-4}$ & 0.0036 \\
\hline & Best best-so-far & 0.0110 & $6.87 \times 10^{-4}$ & $3.16 \times 10^{-20}$ & $9.82 \times 10^{-6}$ & $1.69 \times 10^{-17}$ \\
\hline & Std best-so-far & 0.0352 & 0.3164 & 0.1134 & $8.55 \times 10^{-4}$ & 0.0287 \\
\hline \multirow{4}{*}{$F_{13}$} & Average best-so-far & 0.0817 & 0 & 0 & 0.0477 & 0 \\
\hline & Median best-so-far & 0.0325 & 0 & 0 & 0.0458 & 0 \\
\hline & Best best-so-far & $1.41 \times 10^{-8}$ & 0 & 0 & 0.0073 & 0 \\
\hline & Std best-so-far & 0.1074 & 0 & 0 & 0.0447 & 0 \\
\hline
\end{tabular}

Table 6. Minimization result of benchmark functions in Table 3 with $t_{\text {Max }}=500$.

\begin{tabular}{|c|c|c|c|c|c|c|}
\hline Functions & & RGA & $\mathrm{PSO}$ & SGSA & DGSA & BH-GSA \\
\hline \multirow{2}{*}{$F_{14}$} & Average best-so-far & 0.9980 & 0.9980 & 4.7206 & 0.9980 & 0.9980 \\
\hline & Median best-so-far & 0.9980 & 0.9980 & 3.3010 & 0.9980 & 0.9980 \\
\hline \multirow{2}{*}{$n=2$} & Best best-so-far & 0.9980 & 0.9980 & 0.9980 & 0.9980 & 0.9980 \\
\hline & Std best-so-far & $1.19 \times 10^{-5}$ & $7.14 \times 10^{-17}$ & 3.3401 & $5.18 \times 10^{-9}$ & $8.40 \times 10^{-11}$ \\
\hline \multirow[b]{2}{*}{$F_{15}$} & Average best-so-far & 0.0040 & 0.0028 & 0.0029 & $8.28 \times 10^{-4}$ & 0.0014 \\
\hline & Median best-so-far & 0.0017 & $5.05 \times 10^{-4}$ & 0.0022 & $6.82 \times 10^{-4}$ & $6.93 \times 10^{-3}$ \\
\hline \multirow{2}{*}{$n=4$} & Best best-so-far & 0.0011 & $2.07 \times 10^{-4}$ & 0.0016 & $4.10 \times 10^{-4}$ & $3.52 \times 10^{-4}$ \\
\hline & Std best-so-far & 0.0062 & 0.0069 & 0.0018 & $2.24 \times 10^{-4}$ & $1.23 \times 10^{-4}$ \\
\hline \multirow{2}{*}{$F_{16}$} & Average best-so-far & -1.0316 & -1.0316 & -1.0316 & -1.0282 & -1.0316 \\
\hline & Median best-so-far & -1.0316 & -1.0316 & -1.0316 & -1.0295 & -1.0316 \\
\hline \multirow{2}{*}{$n=2$} & Best best-so-far & -1.0316 & -1.0316 & -1.0316 & -1.0316 & -1.0316 \\
\hline & Std best-so-far & $1.54 \times 10^{-4}$ & $6.09 \times 10^{-17}$ & $3.12 \times 10^{-16}$ & 0.0027 & $6.22 \times 10^{-13}$ \\
\hline
\end{tabular}


Table 6. (Continued)

\begin{tabular}{|c|c|c|c|c|c|c|}
\hline Functions & & RGA & $\mathrm{PSO}$ & SGSA & DGSA & BH-GSA \\
\hline \multirow{2}{*}{$F_{17}$} & Average best-so-far & 0.3996 & 0.3979 & 0.3979 & 0.4031 & 0.3979 \\
\hline & Median best-so-far & 0.3980 & 0.3979 & 0.3979 & 0.4009 & 0.3979 \\
\hline \multirow[t]{2}{*}{$n=2$} & Best best-so-far & 0.3979 & 0.3979 & 0.3979 & 0.3979 & 0.3979 \\
\hline & Std best-so-far & 0.0048 & 0 & 0 & 0.0064 & 0 \\
\hline \multirow{2}{*}{$F_{18}$} & Average best-so-far & 5.7045 & 3.0000 & 3.0000 & 3.3988 & 3.0000 \\
\hline & Median best-so-far & 3.0005 & 3.0000 & 3.0000 & 3.2974 & 3.0000 \\
\hline \multirow{2}{*}{$n=2$} & Best best-so-far & 3.0000 & 3.0000 & 3.0000 & 3.0211 & 3.0000 \\
\hline & Std best-so-far & 8.5399 & $3.11 \times 10^{-15}$ & $8.74 \times 10^{-15}$ & 0.3684 & $6.88 \times 10^{-15}$ \\
\hline \multirow{2}{*}{$F_{19}$} & Average best-so-far & -3.8627 & -3.8628 & -3.8628 & -3.8161 & -3.8628 \\
\hline & Median best-so-far & -3.8628 & -3.8628 & -3.8628 & -3.8277 & -3.8628 \\
\hline \multirow{2}{*}{$n=3$} & Best best-so-far & -3.8628 & -3.8628 & -3.8628 & -3.8586 & -3.8628 \\
\hline & Std best-so-far & $2.01 \times 10^{-4}$ & $4.98 \times 10^{-16}$ & $5.23 \times 10^{-15}$ & 0.0347 & $1.69 \times 10^{-15}$ \\
\hline \multirow{2}{*}{$F_{20}$} & Average best-so-far & -3.3099 & -3.2369 & -3.3220 & -3.1641 & -3.3220 \\
\hline & Median best-so-far & -3.3217 & -3.2031 & -3.3220 & -3.1738 & -3.3220 \\
\hline \multirow{2}{*}{$n=6$} & Best best-so-far & -3.3220 & -3.3220 & -3.3220 & -3.2718 & -3.3220 \\
\hline & Std best-so-far & 0.0375 & 0.0773 & $8.18 \times 10^{-15}$ & 0.0577 & $9.72 \times 10^{-16}$ \\
\hline \multirow{2}{*}{$F_{21}$} & Average best-so-far & -5.6605 & -6.6290 & -9.9541 & -10.1532 & -10.1532 \\
\hline & Median best-so-far & -2.6824 & -5.1008 & -10.1486 & -10.1532 & -10.1532 \\
\hline \multirow{2}{*}{$n=4$} & Best best-so-far & -10.152 & -10.1532 & -10.1532 & -10.1532 & -10.1532 \\
\hline & Std best-so-far & 3.8581 & 3.1701 & 0.5324 & $6.77 \times 10^{-5}$ & $2.75 \times 10^{-5}$ \\
\hline \multirow{2}{*}{$F_{22}$} & Average best-so-far & -7.3421 & -9.1118 & -10.4008 & -10.4028 & -10.4029 \\
\hline & Median best-so-far & -10.3932 & -10.4029 & -10.4029 & -10.4028 & -10.4029 \\
\hline \multirow{2}{*}{$n=4$} & Best best-so-far & -10.4029 & -10.4029 & -10.4029 & -10.4029 & -10.4029 \\
\hline & Std best-so-far & 3.9440 & 2.7783 & 0.0120 & $3.85 \times 10^{-5}$ & $2.58 \times 10^{-5}$ \\
\hline \multirow{2}{*}{$F_{23}$} & Average best-so-far & -6.2541 & -9.7634 & -10.5364 & -10.5363 & -10.5364 \\
\hline & Median best-so-far & -4.5054 & -10.5364 & -10.5364 & -10.5363 & -10.5364 \\
\hline \multirow[b]{2}{*}{$n=4$} & Best best-so-far & -10.5364 & -10.536 & -10.5364 & -10.5364 & -10.5364 \\
\hline & Std best-so-far & 3.7500 & 2.4444 & $1.65 \times 10^{-15}$ & $2.26 \times 10^{-5}$ & $8.35 \times 10^{-5}$ \\
\hline
\end{tabular}

Table 7. Wilcoxon signed ranks test results for unimodal test functions of set 1 .

\begin{tabular}{lllll}
\hline Comparison & $R^{+}$ & $R^{-}$ & $z$-value & $p$-value \\
\hline BH-GSA versus RGA & 28 & 0 & 2.36643 & 0.01796 \\
BH-GSA versus PSO & 28 & 0 & 2.36643 & 0.01796 \\
BH-GSA versus GSA & 15 & 0 & 2.02259 & 0.04311 \\
BH-GSA versus DGSA & 1 & 2 & 0.44721 & 0.65472 \\
\hline
\end{tabular}

Table 8. Wilcoxon signed ranks test results for multimodal test functions of set 1 .

\begin{tabular}{lllll}
\hline Comparison & $R^{+}$ & $R^{-}$ & $z$-value & $p$-value \\
\hline BH-GSA versus RGA & 85 & 20 & 2.04023 & 0.04132 \\
BH-GSA versus PSO & 41 & 14 & 1.37604 & 0.16880 \\
BH-GSA versus GSA & 50 & 5 & 2.29341 & 0.02182 \\
BH-GSA versus DGSA & 47 & 19 & 1.24475 & 0.21322 \\
\hline
\end{tabular}


Table 9. Average ranking of algorithms based on the average best-so-far for unimodal test functions of set 1 .

\begin{tabular}{llllll}
\hline Algorithm & RGA & PSO & GSA & DGSA & BH-GSA \\
\hline Ranking & 4.71 & 4.14 & 2.64 & 1.86 & 1.64 \\
\hline
\end{tabular}

Table 10. Results of Friedman's test based on the average best-so-far for unimodal test functions of set 1.

\begin{tabular}{lll}
\hline Method & Statistical value & $p$-value \\
\hline \multirow{2}{*}{ Friedman } & 22.50381 & 0.00015 \\
\hline
\end{tabular}

Table 11. Average ranking of algorithms based on the average best-so-far for multimodal test functions of set 1 .

\begin{tabular}{llllll}
\hline Algorithm & RGA & PSO & GSA & DGSA & BH-GSA \\
\hline Ranking & 3.88 & 3.19 & 3.06 & 2.78 & 2.09 \\
\hline
\end{tabular}

Table 12. Results of Friedman's test based on the average best-so-far for multimodal test functions of set 1 .

\begin{tabular}{llc}
\hline Method & Statistical value & $p$-value \\
\hline Friedman & 12.28673 & 0.01534 \\
\hline
\end{tabular}

Table 13. The CEC 2005 benchmark functions.

\begin{tabular}{|l|}
\hline Unimodal Functions: \\
$F_{1}:$ Shifted Sphere Function \\
$F_{2}:$ Shifted Schwefel's Problem 1.2 \\
$F_{3}:$ Shifted Rotated High Conditioned Elliptic Function \\
$F_{4}:$ Shifted Schwefel's Problem 1.2 with Noise in Fitness \\
$F_{5}:$ Schwefel's Problem 2.6 with Global Optimum on Bounds \\
\hline Multimodol Functions: \\
$F_{6}:$ Shifted Rosenbrock's Function \\
$F_{7}:$ Shifted Rotated Griewank's Function without Bounds \\
$F_{8}:$ Shifted Rotated Ackley's Function \\
with Global Optimum on Bounds \\
$F_{9}:$ Shifted Rastrigin's Function \\
$F_{10}:$ Shifted Rotated Rastrigin's Function \\
$F_{11}:$ Shifted Rotated Weierstrass Function \\
$F_{12}:$ Schwefel's Problem 2.13 \\
$F_{13}:$ Expanded Extended Griewank's \\
plus Rosenbrock's Function (F8F2) \\
$F_{14}:$ Shifted Rotated Expanded Scaffer's F6 \\
$F_{15}:$ Hybrid Composition Function \\
$F_{16}:$ Rotated Hybrid Composition Function \\
$F_{17}:$ Rotated Hybrid Composition Function with Noise in Fitnes \\
$F_{18}:$ Rotated Hybrid Composition Function \\
$F_{19}:$ Rotated Hybrid Composition Function \\
with a Narrow Basin for the Global Optimum \\
$F_{20}:$ Rotated Hybrid Composition Function \\
with the Global Optimum on the Bounds \\
$F_{21}:$ Rotated Hybrid Composition Function \\
$F_{22}:$ Rotated Hybrid Composition Function \\
with High Condition Number Matrix \\
$F_{23}:$ Non-Continuous Rotated Hybrid Composition Function \\
$F_{24}:$ Rotated Hybrid Composition Function \\
$F_{25}:$ Rotated Hybrid Composition Function without Bounds \\
\hline
\end{tabular}


Table 14. The comparison of BH-GSA with other algorithms $(n=10)$.

\begin{tabular}{|c|c|c|c|c|c|}
\hline Func & $\mathrm{ES}^{43}$ & PLES $^{43}$ & DGSA $^{28}$ & QPSO $^{44}$ & BH-GSA \\
\hline$F_{1}$ & $8.16 \times 10^{-9}$ & $8.40 \times 10^{-9}$ & $1.13 \times 10^{+4}$ & $\mathbf{0}$ & $6.82 \times 10^{-14}$ \\
\hline$F_{2}$ & $2.90 \times 10^{-5}$ & $9.65 \times 10^{-9}$ & $1.27 \times 10^{+4}$ & $\mathbf{0}$ & $1.87 \times 10^{-13}$ \\
\hline$F_{3}$ & $3.52 \times 10^{+5}$ & $1.18 \times 10^{+5}$ & $1.19 \times 10^{+8}$ & $8.07 \times 10^{+4}$ & $8.89 \times 10^{+4}$ \\
\hline$F_{4}$ & $4.13 \times 10^{+3}$ & $6.03 \times 10^{+3}$ & $1.53 \times 10^{+4}$ & 0 & $2.25 \times 10^{-13}$ \\
\hline$F_{5}$ & $1.36 \times 10^{+3}$ & $9.05 \times 10^{+2}$ & $1.65 \times 10^{+4}$ & 0 & 85.0937 \\
\hline$F_{6}$ & $7.49 \times 10^{+1}$ & $3.05 \times 10^{+1}$ & $2.00 \times 10^{+9}$ & 4.5347 & 1.6933 \\
\hline$F_{7}$ & 1.1826 & 4.0943 & $1.26 \times 10^{+3}$ & 636.9838 & $1.29 \times 10^{+3}$ \\
\hline$F_{8}$ & $2.03 \times 10^{+1}$ & $2.03 \times 10^{+1}$ & 20.2713 & 20.2671 & 20.0074 \\
\hline$F_{9}$ & $5.62 \times 10^{+1}$ & $2.62 \times 10^{+1}$ & 64.4160 & 1.9932 & 3.5421 \\
\hline$F_{10}$ & $1.18 \times 10^{+2}$ & $3.66 \times 10^{+1}$ & 90.7660 & 9.0177 & 3.1043 \\
\hline$F_{11}$ & $1.14 \times 10^{+1}$ & 9.9390 & 9.7992 & 8.7431 & $6.90 \times 10^{-5}$ \\
\hline$F_{12}$ & $7.36 \times 10^{+4}$ & $1.37 \times 10^{+4}$ & $6.32 \times 10^{+4}$ & $3.80 \times 10^{+4}$ & 129.3648 \\
\hline$F_{13}$ & $1.71 \times 10^{+4}$ & $3.00 \times 10^{+1}$ & 6.1318 & 0.5358 & 1.2063 \\
\hline$F_{14}$ & 4.1490 & 4.1802 & 4.3965 & 3.5215 & 4.2609 \\
\hline$F_{15}$ & $7.48 \times 10^{+2}$ & $4.77 \times 10^{+2}$ & 715.4225 & 413.1732 & 204.0584 \\
\hline$F_{16}$ & $5.31 \times 10^{+2}$ & $1.81 \times 10^{+2}$ & 387.9110 & $1.44 \times 10^{+3}$ & 52.6582 \\
\hline$F_{17}$ & $4.49 \times 10^{+2}$ & $1.95 \times 10^{+2}$ & 448.6294 & $1.34 \times 10^{+3}$ & 62.5762 \\
\hline$F_{18}$ & $1.14 \times 10^{+3}$ & $1.01 \times 10^{+3}$ & $1.03 \times 10^{+3}$ & $1.97 \times 10^{+3}$ & 839.2796 \\
\hline$F_{19}$ & $1.12 \times 10^{+3}$ & $1.00 \times 10^{+3}$ & $1.13 \times 10^{+3}$ & $1.96 \times 10^{+3}$ & 856.0262 \\
\hline$F_{20}$ & $1.31 \times 10^{+3}$ & $9.98 \times 10^{+2}$ & $1.25 \times 10^{+3}$ & $1.97 \times 10^{+3}$ & 860.0096 \\
\hline$F_{21}$ & $1.32 \times 10^{+3}$ & $1.07 \times 10^{+3}$ & $1.36 \times 10^{+3}$ & $2.04 \times 10^{+3}$ & 800 \\
\hline$F_{22}$ & $9.29 \times 10^{+2}$ & $8.80 \times 10^{+2}$ & $1.10 \times 10^{+3}$ & $1.63 \times 10^{+3}$ & 715.9475 \\
\hline$F_{23}$ & $1.34 \times 10^{+3}$ & $1.11 \times 10^{+3}$ & $1.36 \times 10^{+3}$ & $2.29 \times 10^{+3}$ & 970.5031 \\
\hline$F_{24}$ & $1.19 \times 10^{+3}$ & $2.82 \times 10^{+2}$ & $1.33 \times 10^{+3}$ & $1.10 \times 10^{+3}$ & 276 \\
\hline$F_{25}$ & $4.15 \times 10^{+2}$ & $6.92 \times 10^{+2}$ & $1.42 \times 10^{+3}$ & $1.12 \times 10^{+3}$ & $1.30 \times 10^{+3}$ \\
\hline
\end{tabular}

Table 15. Wilcoxon signed ranks test results for unimodal test functionsof set 2 .

\begin{tabular}{lllll}
\hline Comparison & $R^{+}$ & $R^{-}$ & $z$-value & $p$-value \\
\hline BH-GSA versus DGSA & 15 & 0 & 2.02259 & 0.04311 \\
BH-GSA versus ES & 15 & 0 & 2.02259 & 0.04311 \\
BH-GSA versus PLES & 15 & 0 & 2.02259 & 0.04311 \\
BH-GSA versus QPSO & 0 & 15 & 2.02259 & 0.04311 \\
\hline
\end{tabular}

Table 16. Average ranking of algorithms based on the average best-so-far for unimodal test functions of set 2 .

\begin{tabular}{llllll}
\hline Algorithm & DGSA & ES & PLES & QPSO & BH-GSA \\
\hline Ranking & 5.00 & 3.60 & 3.40 & 1.00 & 2.00 \\
\hline
\end{tabular}

Table 17. Results of Friedman's test based on the average best-so-far for unimodal test functions of set 2 .

\begin{tabular}{lll}
\hline Method & Statistical value & $p$-value \\
\hline Friedman & 19.040 & 0.001 \\
\hline
\end{tabular}


Table 18. Wilcoxon signed ranks test results for multimodal test functions of set 2 .

\begin{tabular}{lllll}
\hline Comparison & $R^{+}$ & $R^{-}$ & $z$-value & $p$-value \\
\hline BH-GSA versus DGSA & 205 & 5 & 3.73326 & 0.00018 \\
BH-GSA versus ES & 175 & 35 & 2.61328 & 0.00896 \\
BH-GSA versus PLES & 191 & 19 & 3.21060 & 0.00132 \\
BH-GSA versus QPSO & 183 & 27 & 2.91194 & 0.00454 \\
\hline
\end{tabular}

Table 19. Average ranking of algorithms based on the average best-so-far for multimodal test functions of set 2 .

\begin{tabular}{cccccc}
\hline Algorithm & DGSA & ES & PLES & QPSO & BH-GSA \\
\hline \multirow{2}{*}{ Ranking } & 3.85 & 3.68 & 2.73 & 3.20 & 1.55 \\
\hline
\end{tabular}

Table 20. Results of Friedman's test based on the average best-so-far for multimodal test functions of set 2 .

\begin{tabular}{lll}
\hline Method & Statistical value & $p$-value \\
\hline Friedman & 27.23809 & 0.00002 \\
\hline
\end{tabular}

Table 21. Error values achieved at FEs $=1 \mathrm{e}+3,1 \mathrm{e}+4,1 \mathrm{e}+5$ for functions of CEC 2005(n=10).

\begin{tabular}{|c|c|c|c|c|c|c|}
\hline \multicolumn{2}{|c|}{ FES Func } & 1 & 2 & 3 & 4 & 5 \\
\hline \multirow{7}{*}{$1 \mathrm{e}+3$} & $1^{\text {th }}$ & 875.7649 & $5.62 \times 10^{+3}$ & $1.36 \times 10^{+7}$ & $1.07 \times 10^{+4}$ & $9.71 \times 10^{+3}$ \\
\hline & $7^{\text {th }}$ & $1.11 \times 10^{+3}$ & $5.67 \times 10^{+3}$ & $2.03 \times 10^{+7}$ & $1.11 \times 10^{+4}$ & $1.12 \times 10^{+4}$ \\
\hline & $13^{\text {th }}$ & $2.43 \times 10^{+3}$ & $6.55 \times 10^{+3}$ & $3.77 \times 10^{+7}$ & $1.62 \times 10^{+4}$ & $1.60 \times 10^{+4}$ \\
\hline & $19^{\text {th }}$ & $3.56 \times 10^{+3}$ & $7.95 \times 10^{+3}$ & $5.06 \times 10^{+7}$ & $1.95 \times 10^{+4}$ & $1.66 \times 10^{+4}$ \\
\hline & $25^{\text {th }}$ & $4.69 \times 10^{+3}$ & $8.98 \times 10^{+3}$ & $6.77 \times 10^{+7}$ & $2.31 \times 10^{+4}$ & $1.73 \times 10^{+4}$ \\
\hline & Mean & $2.67 \times 10^{+3}$ & $7.08 \times 10^{+3}$ & $3.73 \times 10^{+7}$ & $1.69 \times 10^{+4}$ & $1.53 \times 10^{+4}$ \\
\hline & Std & $1.12 \times 10^{+3}$ & 988.6752 & $1.27 \times 10^{+7}$ & $3.02 \times 10^{+3}$ & $1.78 \times 10^{+3}$ \\
\hline \multirow{7}{*}{$1 \mathrm{e}+4$} & $1^{\text {th }}$ & $5.68 \times 10^{-14}$ & 411.9321 & $1.92 \times 10^{+5}$ & $9.58 \times 10^{+3}$ & $6.74 \times 10^{+3}$ \\
\hline & $7^{\text {th }}$ & $7.77 \times 10^{-14}$ & 637.7552 & $2.75 \times 10^{+5}$ & $1.26 \times 10^{+4}$ & $7.56 \times 10^{+3}$ \\
\hline & $13^{\text {th }}$ & $8.36 \times 10^{-14}$ & 783.6008 & $4.24 \times 10^{+5}$ & $1.36 \times 10^{+4}$ & $8.23 \times 10^{+3}$ \\
\hline & $19^{\text {th }}$ & $1.03 \times 10^{-13}$ & 883.8554 & $8.33 \times 10^{+5}$ & $1.48 \times 10^{+4}$ & $8.51 \times 10^{+3}$ \\
\hline & $25^{\text {th }}$ & $2.27 \times 10^{-13}$ & $1.32 \times 10^{+3}$ & $9.06 \times 10^{+5}$ & $1.69 \times 10^{+4}$ & $9.86 \times 10^{+3}$ \\
\hline & Mean & $1.02 \times 10^{-13}$ & 807.4038 & $5.86 \times 10^{+5}$ & $1.38 \times 10^{+4}$ & $8.45 \times 10^{+3}$ \\
\hline & Std & $5.18 \times 10^{-14}$ & 258.4901 & $2.07 \times 10^{+5}$ & $1.80 \times 10^{+3}$ & 733.8931 \\
\hline \multirow{7}{*}{$1 \mathrm{e}+5$} & $1^{\text {th }}$ & 0 & $2.84 \times 10^{-13}$ & $2.26 \times 10^{+4}$ & $5.68 \times 10^{-14}$ & 28.9127 \\
\hline & $7^{\text {th }}$ & $1.13 \times 10^{-14}$ & $5.68 \times 10^{-13}$ & $2.63 \times 10^{+4}$ & $1.70 \times 10^{-13}$ & 34.8234 \\
\hline & $13^{\text {th }}$ & $5.68 \times 10^{-14}$ & $1.76 \times 10^{-12}$ & $5.68 \times 10^{+4}$ & $2.27 \times 10^{-13}$ & 52.5562 \\
\hline & $19^{\text {th }}$ & $3.43 \times 10^{-13}$ & $2.04 \times 10^{-12}$ & $8.70 \times 10^{+4}$ & $3.41 \times 10^{-13}$ & 101.9107 \\
\hline & $25^{\text {th }}$ & $5.73 \times 10^{-13}$ & $6.82 \times 10^{-12}$ & $2.58 \times 10^{+5}$ & $4.54 \times 10^{-14}$ & 260.5208 \\
\hline & Mean & $6.82 \times 10^{-14}$ & $1.87 \times 10^{-13}$ & $8.89 \times 10^{+4}$ & $2.25 \times 10^{-13}$ & 85.0937 \\
\hline & Std & $3.28 \times 10^{-14}$ & $3.12 \times 10^{-14}$ & $5.44 \times 10^{+4}$ & $1.14 \times 10^{-13}$ & 52.8263 \\
\hline
\end{tabular}


Table 21. (Continued)

\begin{tabular}{|c|c|c|c|c|c|c|}
\hline \multicolumn{2}{|c|}{ FES Func } & 6 & 7 & 8 & 9 & 10 \\
\hline \multirow{7}{*}{$1 e+3$} & $1^{\text {th }}$ & $3.65 \times 10^{+7}$ & $1.27 \times 10^{+3}$ & 20.3011 & 2.9894 & 1.9899 \\
\hline & $7^{\text {th }}$ & $5.57 \times 10^{+7}$ & $1.32 \times 10^{+3}$ & 20.3896 & 3.9798 & 2.9849 \\
\hline & $13^{\text {th }}$ & $1.12 \times 10^{+8}$ & $1.41 \times 10^{+3}$ & 20.4987 & 4.9748 & 3.9748 \\
\hline & $19^{\text {th }}$ & $1.85 \times 10^{+8}$ & $1.69 \times 10^{+3}$ & 20.6323 & 8.9546 & 4.9798 \\
\hline & $25^{\text {th }}$ & $3.46 \times 10^{+8}$ & $1.85 \times 10^{+3}$ & 20.6504 & 9.9496 & 7.9597 \\
\hline & Mean & $1.89 \times 10^{+8}$ & $1.45 \times 10^{+3}$ & 20.4923 & 5.9698 & 4.6564 \\
\hline & Std & $8.12 \times 10^{+7}$ & 139.3609 & 0.0961 & 2.2978 & 1.6679 \\
\hline \multirow{7}{*}{$1 \mathrm{e}+4$} & $1^{\text {th }}$ & 7.6077 & $1.27 \times 10^{+3}$ & 20.1666 & 1.9899 & 0 \\
\hline & $7^{\text {th }}$ & 7.6528 & $1.31 \times 10^{+3}$ & 20.2137 & 2.9848 & 0.9949 \\
\hline & $13^{\text {th }}$ & 7.8996 & $1.62 \times 10^{+3}$ & 20.3371 & 3.3349 & 2.9848 \\
\hline & $19^{\text {th }}$ & 76.1645 & $1.64 \times 10^{+3}$ & 20.3923 & 4.9747 & 3.9598 \\
\hline & $25^{\text {th }}$ & 141.1563 & $1.87 \times 10^{+3}$ & 20.4606 & 6.9647 & 3.9748 \\
\hline & Mean & 52.2542 & $1.47 \times 10^{+3}$ & 20.4138 & 4.0594 & 3.0247 \\
\hline & Std & 174.7530 & 158.7508 & 0.0873 & 1.3751 & 1.4780 \\
\hline \multirow{7}{*}{$1 \mathrm{e}+5$} & $1^{\text {th }}$ & 1.2432 & $1.26 \times 10^{+3}$ & 20.0003 & 0.9940 & 0 \\
\hline & $7^{\text {th }}$ & 1.3991 & $1.27 \times 10^{+3}$ & 20.0005 & 1.9899 & 0.9950 \\
\hline & $13^{\text {th }}$ & 1.6070 & $1.28 \times 10^{+3}$ & 20.0018 & 2.9849 & 2.9849 \\
\hline & $19^{\text {th }}$ & 1.6403 & $1.30 \times 10^{+3}$ & 20.0175 & 2.9858 & 3.9798 \\
\hline & $25^{\text {th }}$ & 2.1560 & $1.38 \times 10^{+3}$ & 20.0299 & 4.9740 & 5.9697 \\
\hline & Mean & 1.6933 & $1.29 \times 10^{+3}$ & 20.0074 & 3.5421 & 3.1043 \\
\hline & Std & 0.2314 & 30.1695 & 0.0095 & 1.2871 & 1.6329 \\
\hline
\end{tabular}

Table 21. (Continued)

\begin{tabular}{|c|c|c|c|c|c|c|}
\hline \multicolumn{2}{|c|}{$\begin{array}{l}\text { FES } \\
\text { Func }\end{array}$} & 11 & 12 & 13 & 14 & 15 \\
\hline \multirow{7}{*}{$1 e+3$} & $1^{\text {th }}$ & 0.0710 & $1.00 \times 10^{+3}$ & 0.4926 & 4.1984 & 127.5608 \\
\hline & $7^{\text {th }}$ & 0.0872 & $1.31 \times 10^{+3}$ & 1.0579 & 4.2591 & 157.9742 \\
\hline & $13^{\text {th }}$ & 0.9029 & $2.26 \times 10^{+3}$ & 1.3974 & 4.5252 & 400 \\
\hline & $19^{\text {th }}$ & 1.8075 & $2.33 \times 10^{+3}$ & 1.4170 & 4.5899 & 400 \\
\hline & $25^{\text {th }}$ & 2.3480 & $5.13 \times 10^{+3}$ & 2.5753 & 4.6094 & 458.3054 \\
\hline & Mean & 1.0444 & $1.47 \times 10^{+3}$ & 1.4934 & 4.4574 & 212.1892 \\
\hline & Std & 0.7687 & $1.69 \times 10^{+3}$ & 0.5273 & 0.1011 & 142.6050 \\
\hline \multirow{7}{*}{$1 \mathrm{e}+4$} & $1^{\text {th }}$ & $7.04 \times 10^{-5}$ & 18.2253 & 1.3092 & 3.9195 & 0 \\
\hline & $7^{\text {th }}$ & $1.69 \times 10^{-4}$ & 25.7388 & 1.7555 & 4.3359 & 37.6241 \\
\hline & $13^{\text {th }}$ & 0.1158 & 217.2541 & 2.0906 & 4.3890 & 46.9651 \\
\hline & $19^{\text {th }}$ & 1.0556 & $1.02 \times 10^{+3}$ & 2.1866 & 4.4173 & 89.7831 \\
\hline & $25^{\text {th }}$ & 1.6545 & $6.04 \times 10^{+3}$ & 2.2214 & 4.4311 & 439.6124 \\
\hline & Mean & 0.1421 & $1.79 \times 10^{+3}$ & 1.7764 & 4.3986 & 342.8198 \\
\hline & Std & 0.3872 & $1.90 \times 10^{+3}$ & 0.4698 & 0.1412 & 167.4221 \\
\hline \multirow{7}{*}{$1 \mathrm{e}+5$} & $1^{\text {th }}$ & $4.98 \times 10^{-5}$ & $2.10 \times 10^{-12}$ & 0.7561 & 3.9770 & 0 \\
\hline & $7^{\text {th }}$ & $5.12 \times 10^{-5}$ & 3.2206 & 1.1212 & 4.0987 & $1.4210 \mathrm{e}-14$ \\
\hline & $13^{\text {th }}$ & $7.25 \times 10^{-5}$ & 3.3904 & 1.4955 & 4.2049 & 94.4594 \\
\hline & $19^{\text {th }}$ & $8.25 \times 10^{-5}$ & 3.8613 & 1.6009 & 4.3745 & 400.0000 \\
\hline & $25^{\text {th }}$ & $9.24 \times 10^{-5}$ & $1.24 \times 10^{+3}$ & 1.7013 & 4.5046 & 447.5146 \\
\hline & Mean & $6.90 \times 10^{-5}$ & 129.3648 & 1.2063 & 4.2609 & 204.0584 \\
\hline & Std & $1.17 \times 10^{-5}$ & 429.4598 & 0.2424 & 0.1397 & 175.6590 \\
\hline
\end{tabular}


Table 21. (Continued)

\begin{tabular}{|c|c|c|c|c|c|c|}
\hline \multicolumn{2}{|c|}{ FES Func } & 16 & 17 & 18 & 19 & 20 \\
\hline \multirow{7}{*}{$1 \mathrm{e}+3$} & $1^{\text {th }}$ & 99.3038 & 98.4976 & 900.0622 & 900.0000 & 900.5337 \\
\hline & $7^{\text {th }}$ & 103.9934 & 109.1906 & 901.5932 & 908.6740 & 903.7859 \\
\hline & $13^{\text {th }}$ & 106.2353 & 116.6630 & 928.9229 & 915.2858 & 935.3063 \\
\hline & $19^{\text {th }}$ & 113.0868 & 121.7023 & 956.8357 & 917.9505 & 941.5988 \\
\hline & $25^{\text {th }}$ & 124.1496 & 128.1565 & 962.2479 & 919.4297 & 966.5125 \\
\hline & Mean & 105.4861 & 110.5328 & 916.0996 & 905.1901 & 909.1597 \\
\hline & Std & 12.7720 & 13.6899 & 21.7478 & 5.5027 & 29.1454 \\
\hline \multirow{7}{*}{$1 \mathrm{e}+4$} & $1^{\text {th }}$ & 67.3031 & 60.6844 & 900.0276 & 900.0059 & 800.0000 \\
\hline & $7^{\text {th }}$ & 82.0207 & 93.6799 & 901.5180 & 900.0646 & 900.0059 \\
\hline & $13^{\text {th }}$ & 94.2556 & 95.6312 & 905.1937 & 900.0748 & 900.0743 \\
\hline & $19^{\text {th }}$ & 97.8406 & 98.6143 & 909.9466 & 901.5510 & 902.4812 \\
\hline & $25^{\text {th }}$ & 109.4240 & 111.5311 & 912.5071 & 925.4205 & 906.3941 \\
\hline & Mean & 97.1861 & 99.0728 & 903.0020 & 904.2967 & 877.1554 \\
\hline & Std & 9.1912 & 9.6105 & 3.4793 & 6.1606 & 44.2763 \\
\hline \multirow{7}{*}{$1 \mathrm{e}+5$} & $1^{\text {th }}$ & $1.42 \times 10^{-14}$ & 0 & 800 & 800 & 800 \\
\hline & $7^{\text {th }}$ & 61.4401 & 60.2966 & 800 & 800 & 800 \\
\hline & $13^{\text {th }}$ & 72.1220 & 81.1362 & 800 & 800 & 900.8918 \\
\hline & $19^{\text {th }}$ & 90.6816 & 86.3142 & 900.0011 & 800 & 904.8482 \\
\hline & $25^{\text {th }}$ & 99.9401 & 88.7401 & 900.6474 & 900.2719 & 915.8060 \\
\hline & Mean & 52.6582 & 62.5762 & 839.2796 & 856.0262 & 860.0096 \\
\hline & Std & 42.6980 & 45.5680 & 53.9551 & 50.6860 & 50.0080 \\
\hline
\end{tabular}

Table 21. (Continued)

\begin{tabular}{|c|c|c|c|c|c|c|}
\hline \multicolumn{2}{|c|}{ FES Func } & 21 & 22 & 23 & 24 & 25 \\
\hline \multirow{7}{*}{$1 e+3$} & $1^{\text {th }}$ & 800.0000 & 752.5243 & 970.5031 & 200 & $1.28 \times 10^{+3}$ \\
\hline & $7^{\text {th }}$ & 841.7514 & 755.3251 & $1.04 \times 10^{+3}$ & $1.15 \times 10^{+3}$ & $1.33 \times 10^{+3}$ \\
\hline & $13^{\text {th }}$ & 910.5146 & 800.0000 & $1.08 \times 10^{+3}$ & $1.17 \times 10^{+3}$ & $1.35 \times 10^{+3}$ \\
\hline & $19^{\text {th }}$ & $1.15 \times 10^{+3}$ & 800.0000 & $1.12 \times 10^{+3}$ & $1.22 \times 10^{+3}$ & $1.36 \times 10^{+3}$ \\
\hline & $25^{\text {th }}$ & $1.21 \times 10^{+3}$ & 800.0000 & $1.24 \times 10^{+3}$ & $1.27 \times 10^{+3}$ & $1.37 \times 10^{+3}$ \\
\hline & Mean & 911.6849 & 789.8992 & $1.09 \times 10^{+3}$ & $1.16 \times 10^{+3}$ & $1.34 \times 10^{+3}$ \\
\hline & Std & 184.9839 & 30.6024 & 85.7020 & 205.9180 & 17.5358 \\
\hline \multirow{7}{*}{$1 \mathrm{e}+4$} & $1^{\text {th }}$ & 300 & 743.3557 & 970.5031 & 200 & $1.30 \times 10^{+3}$ \\
\hline & $7^{\text {th }}$ & 800 & 762.1394 & 970.5031 & $1.09 \times 10^{+3}$ & $1.31 \times 10^{+3}$ \\
\hline & $13^{\text {th }}$ & 800 & 777.5103 & 970.5031 & $1.16 \times 10^{+3}$ & $1.32 \times 10^{+3}$ \\
\hline & $19^{\text {th }}$ & 800 & 874.4739 & $1.05 \times 10^{+3}$ & $1.18 \times 10^{+3}$ & $1.33 \times 10^{+3}$ \\
\hline & $25^{\text {th }}$ & 800 & 943.2373 & $1.08 \times 10^{+3}$ & $1.27 \times 10^{+3}$ & $1.35 \times 10^{+3}$ \\
\hline & Mean & 780 & 803.4792 & 997.4094 & $1.11 \times 10^{+3}$ & $1.32 \times 10^{+3}$ \\
\hline & Std & 100 & 47.2459 & 49.1928 & 281.4502 & 10.7267 \\
\hline \multirow{7}{*}{$1 \mathrm{e}+5$} & $1^{\text {th }}$ & 800 & 592.4100 & 970.5031 & 200 & $1.22 \times 10^{+3}$ \\
\hline & $7^{\text {th }}$ & 800 & 659.3091 & 970.5031 & 200 & $1.27 \times 10^{+3}$ \\
\hline & $13^{\text {th }}$ & 800 & 681.6107 & 970.5031 & 200 & $1.29 \times 10^{+3}$ \\
\hline & $19^{\text {th }}$ & 800 & 741.3234 & 970.5031 & 500 & $1.30 \times 10^{+3}$ \\
\hline & $25^{\text {th }}$ & 800 & 742.1079 & 970.5031 & 900 & $1.31 \times 10^{+3}$ \\
\hline & Mean & 800 & 715.9475 & 970.5031 & 276 & $1.30 \times 10^{+3}$ \\
\hline & Std & $\mathbf{0}$ & 46.4624 & $3.4809 \mathrm{e}-013$ & 83.0662 & 19.7972 \\
\hline
\end{tabular}




\section{References}

1. K. S. Tang, K. F. Man, S. Kwong and Q. He, Genetic algorithms and their applications, IEEE Signal Processing Magazine 13 (6) (1996) 22-37.

2. F. V. D. Bergh and A. P. Engelbrecht, A study of particle swarm optimization particle trajectories, Information Sciences, 176(8), pp. 937-971 (2006).

3. J. Kennedy and R. C. Eberhart, Particle swarm optimization, in: Proceedings of IEEE International Conference on Neural Networks, vol. 4, 1995, pp. 19421948.

4. S. Kirkpatrick, C. D. Gelatto and M. P. Vecchi, Optimization by simulated annealing, Science 220 (1983) 671-680.

5. M. Dorigo, V. Maniezzo and A. Colorni, The ant system: optimization by a colony of cooperating agents, IEEE Transactions on Systems, Man, and Cybernetics - Part B 26 (1) (1996) 29-41.

6. O. K. Erol and I. Eksin, A new optimization method: big bang-big crunch, Advances in Engineering Software 37 (2006) 106-111.

7. Ch. Guo, Zh. Jiang, H. Zhang and N. Li, Decompositionbased classified ant colony optimization algorithm for scheduling semiconductor wafer fabrication system, Computers \& Industrial Engineering 62 (2012) 141-151.

8. S. Mondal, A. Bhattacharya and S. H. N. Dey, Multiobjective economic emission load dispatch solution using gravitational search algorithm and considering wind power penetration, Electrical Power and Energy Systems 44(2013) 282-292.

9. D. Hu, A. Sarosh and Y. F. Dong, An improved particle swarm optimizer for parametric optimization of flexible satellite controller, Applied Mathematics and Computation 217 (2011) 8512-8521.

10. T. Ganesan, I. Elamvazuthi, Ku Zilati Ku Shaari and P. Vasant, Swarm intelligence and gravitational search algorithm for multi-objective optimization of synthesis gas production, Applied Energy, 2012 in Press.

11. E. Rashedi, H. Nezamabadi-pour, and S. Saryazdi, Filter modeling using gravitational search algorithm, Engineering Application of Artificial Intelligence, vol. 24, no.1 pp. 117-122, 2011

12. F. Xhafa, A. Barolli, C. Sánchez and L. Barolli, A simulated annealing algorithm for router nodes placement problem in Wireless Mesh Networks, Simulation Modelling Practice and Theory, vol. 19, pp. 2276-2284, 2011.

13. D. d. Serafino, S. Gomez, L. Milano, F. Riccio and G. Toraldo, A genetic algorithm for a global optimization problem arising in the detection of gravitational waves, Springer Science and Business Media, (2010) 48:41-55.

14. P. C. Chang, J. J. Lin and C. H. Liu, An attribute weight assignment and particle swarm optimization algorithm for medical database classifications, Computer Methods and Programs in Biomedicine 107 (2012) 382-392.

15. B. Sahu and D. Mishra, A Novel Feature Selection Algorithm using Particle Swarm Optimization for Cancer
Microarray Data, Procedia Engineering 38 ( 2012 ) 27 31.

16. D. Mishra, Discovery of Overlapping Pattern Biclusters from Gene Expression Data using Hash based PSO, Procedia Technology 4 (2012) 390 - 394.

17. U. Güvença, Y. Sönmezb, S. Dumanc and N. Yörükerend, Combined economic and emission dispatch solution using gravitational search algorithm, Scientia Iranica, 2012 in Press.

18. F. Musharavati and A. M. S. Hamouda, Simulated annealing with auxiliary knowledge for process planning optimization in reconfigurable manufacturing, Robotics and Computer-Integrated Manufacturing 28 (2012) 113131.

19. Q. Zhua, J. Hu and L Henschen, A new moving target interception algorithm for mobile robots based on subgoal forecasting and an improved scout ant algorithm, Applied Soft Computing 13 (2013) 539-549.

20. K. Ioannidis, G. Ch. Sirakoulis and I. Andreadis, Cellular ants: A method to create collision free trajectories for a cooperative robot team, Robotics and Autonomous Systems 59 (2011) 113-127.

21. E. G. Talbi, A taxonomy of hybrid metaheuristics, Journal of Heuristics, 8 (2002) pp. 541-564.

22. Z. Ye, Z. Li and M. Xie, Some improvements on adaptive genetic algorithms for reliability-related applications, Reliability Engineering and System Safety, 95(2), pp. 120-126 (2010).

23. S. H. Ling, H. H. C. Iu, K. Y. Chan, H. K. Lam, B. C. W. Yeung and F. H. Leung, Hybrid Particle Swarm Optimization With Wavelet Mutation and Its Industrial Applications, Systems, Man, and Cybernetics, Part B: Cybernetics, IEEE Transactions on, vol. 38, pp. 743763, June 2008.

24. H. Cheng, N. Xiong, A. V. Vasilakos, L. Tianruo Yang, G. Chen and X. Zhuang, Nodes organization for channel assignment with topology preservation in multi-radio wireless mesh networks, Ad Hoc Networks 10 (2012) 760-773.

25. B. Yu, Z-Z. Yang and B. Yao, An improved ant colony optimization for vehicle routing problem, European Journal of Operational Research, vol. 196, pp. 171176 , July 2009

26. E. Rashedi, H. Nezamabadi-pour and S. Saryazdi, BGSA: binary gravitational search algorithm, Natural Computing, vol.9, no. 3, pp. 727-745, 2010.

27. E. Rashedi, H. Nezamabadi-pour and S. Saryazdi, GSA: a gravitational search algorithm, Information Sciences, 179(13), pp. 2232-2248, 2009.

28. S. Sarafrazi, H. Nezamabadi-pour and S. Saryazdi, Disruption: A new operator in gravitational search algorithm, Scientialranica, pp. 539-548, Feb 2011.

29. C. Li and J. Zhou, Parameters identification of hydrulic turbine governing system using improved gravitational search algorithm, Energy Conversion and Management 52 (2011) 374-381 
30. M. Doraghinejad, H. Nezamabadi-pour and M.M. Farsangi, Black hole: a new operator in gravitational search algorithm for unimodal problems, 20th Iranian Conference on Electrical Engineering (ICEE'12), Tehran, Iran, May 2012 (in Farsi).

31. M. Doraghinejad, H. Nezamabadi-pour, A. H. Sadeghian and M.M.Farsangi, A Hybrid Algorithm Based on Gravitational Search Algorithm for Unimodal Optimization, 2nd International eConference on Computer and Knowledge Engineering (ICCKE'12), Mashhad, Iran, (Oct. 2012), pp. 129-132.

32. A. Hatamlou, Black hole: a new heuristic optimization approach for data clustering, Information Sciences 222 (2013) 175-184

33. M. Markou and S. Singh, Feature selection based on a Black Hole model of data reorganization. In: 17th International conference on pattern recognition (ICPR04), (Cambridge, 2004), pp. 565-568.

34. S. Singh and M. Markou, A black hole novelty detector for video analysis. Pattern Anal Appl, (2005), 8:102114.

35. B. Schutz, A First Course in General Relativity, 2nd edn. (Cambridge University Press, 2009).

36. B. W.Carrol, D. A. Ostlie, An Introduction to Modern Astrophysics, 2nd edn. (Addison Wesley Publishing Company, 2007).
37. N. K. Glendenning, Special and General Relativity, (Springer, 2007)

38. R. A D'Inverno, Introducing Einstein's Relativity, (The United States by Oxford University Press lnc, New York, 1998).

39. X. Yao, Y. Liu and G. Lin, Evolutionary programming made faster, IEEE Transactions on Evolutionary Computation 3 (1999) 82-102.

40. R. L. Haupt and E. Haupt, Practical Genetic Algorithms, 2nd edn. (John Wiley \& Sons, 2004).

41. J. Derrac, S. Garcia, D. Molina and F. Herrera, A practical tutorial on the use of nonparametric statistical tests as a methodology for comparing evolutionary and swarm intelligence algorithms, Swarm and Evolutionary Computation 1 (2011) 3-18.

42. http://www.ntu.edu.sg/home/EPNSugan.

43. L. Costa, A Parameter-less Evolution Strategy for Global Optimization, PhD thesis, Escola de Engenharia, Universidade do Minho, 2005.

44. J. Sun, B. Feng, W. Xu, Particle Swarm Optimization with particles having Quantum Behavior, In Proc. of Congress on Evolutionary Computation, Portland, USA, 2004, pp. $325-331$. 